\title{
Estimates of the Tropospheric Vertical Structure of Neptune Based on Microwave Radiative Transfer Studies
}

\author{
David R. DeBoer and Paul G. Steffes \\ School of Electrical and Computer Engineering, Georgia Institute of Technology, Atlanta, Georgia 30332-0250 \\ E-mail: ddeboer@ee.gatech.edu
}

Received September 25, 1995; revised March 18, 1996

\begin{abstract}
A radiative transfer model incorporating, among other things, the recently measured centimeter wavelength opacity of $\mathrm{H}_{2} \mathrm{~S}$, the full line catalog of $\mathrm{PH}_{3}$, and absorption due to $\mathrm{CO}$ has been developed to study the tropospheric vertical structure of Neptune. To match radio-telescope observations, subsolar amounts of $\mathrm{NH}_{3}$ and supersolar amounts of $\mathrm{H}_{2} \mathrm{~S}$ are found to be needed, as has been previously noted. To match both the measured microwave emission and the measured opacity at 13 $\mathrm{cm}$ and 6.3 bars by Voyager 2 , an $\mathrm{H}_{2} \mathrm{~S}$ dominant atmosphere $\left(\mathrm{H}_{2} \mathrm{~S} / \mathrm{NH}_{3} \approx 40\right)$ with enhanced $\mathrm{PH}_{3}(15 \times$ solar $)$ or $\mathrm{NH}_{3}$ supersaturation with respect to the putative $\mathrm{NH}_{4} \mathrm{SH}$ cloud (400 ppbv) seems to be indicated. Due to the possible importance of $\mathrm{PH}_{3}$ opacity, it is suggested that measurements of its opacity could aid in resolving some of the outstanding ambiguities concerning Neptune's tropospheric structure. 1996 Academic Press, Inc.
\end{abstract}

\section{INTRODUCTION}

Neptune is the most distant of the jovian planets and hence has typically been the last to be scrutinized closely. Voyager 2 made its closest approach to Neptune on August 25,1989 , from which we have learned much of what we know of the planet (see J. Geophys. Rev. 96, 1991). Neptune is a relatively dynamic planet, radiating 2.5 times the amount of energy it receives from the Sun (Conrath et. al. 1989) and exhibiting significant spatial and temporal variability (e.g., Baines and Smith 1990, and references therein).

An analysis of Neptune's microwave spectrum using thermochemical modeling was undertaken by Romani et al. (1989), prior to the Voyager rendezvous, and included opacity from $\mathrm{NH}_{3}, \mathrm{H}_{2} \mathrm{O}$, hydrometeors, and collisionalinduced $\mathrm{H}_{2}$ absorption. Two primary (and related) conclusions from that work are that ammonia seems to be present in subsolar amounts and that the 20 -cm brightness temperature was hard to fit with the rest of the spectrum assuming only thermal emission from the planet. A later analysis (de Pater et al. 1991) of Neptune microwave emission considered some preliminary results from Voyager as well as an estimate of $\mathrm{H}_{2} \mathrm{~S}$ opacity and included new measurements at $20 \mathrm{~cm}$, eliminating much of the difficulty in matching the $20-\mathrm{cm}$ flux. However, $\mathrm{H}_{2} \mathrm{~S}$ opacity was unmeasured at the time, and more complete Voyager results have since been presented. The following analysis of the microwave emission spectrum is the first to include measured $\mathrm{H}_{2} \mathrm{~S}$ parameters as well as the full effects of $\mathrm{PH}_{3}$ and $\mathrm{CO}$.

The primary objective of this analysis is to provide insight into whether hydrogen sulfide is the dominant centimeter wavelength absorber in the upper troposphere (that is, whether the mole fraction of $\mathrm{H}_{2} \mathrm{~S}$ is numerically greater than that of $\mathrm{NH}_{3}$ throughout the atmosphere so that $\mathrm{H}_{2} \mathrm{~S}$ provides the opacity above the $\mathrm{NH}_{4} \mathrm{SH}$ cloud) or if ammonia is the dominant absorber, as well as to begin to understand the importance of phosphine in shaping the microwave spectrum of Neptune. The location and role of sulfur in the giant planets have been identified as key outstanding issues in planetary studies (Space Studies Board 1994), especially for Uranus and Neptune (Lunine 1993). A brief synopsis of the planetary model is presented in the next section, followed by a discussion of the microwave properties of the various constituents and an examination of the constraints used in the models. Modeling results and discussion then follow.

\section{PLANETARY MODEL}

The planetary model consists of two distinct parts, the thermochemical model and the radiative transfer model. The thermochemical model determines the structure of the planet given a set of conditions within the interior, while the radiative transfer model calculates the spectral characteristics of the microwave emission of the planet given the structure from the thermochemical model.

\subsection{Thermochemical Model}

For pressures exceeding 0.5 bar, radiative-convective modeling of jovian-like atmospheres shows that convection should dominate and the temperature-pressure (T-P) pro- 
file should follow an adiabat (Appleby and Hogan 1984, Appleby 1986), which simplifies modeling of the troposphere. Thermochemical modeling computes the temperature-pressure profile and cloud structures assuming an adiabatic atmosphere in hydrostatic equilibrium. The thermochemical model takes an assumed abundance distribution of the atmospheric components deep in the atmosphere (where all constituents are assumed to be gaseous) and steps out in discrete layers of pressure. At each pressure step, $d P$, a check is made to see if any component has condensed out and the new temperature, $T$, is determined. To check for condensation, one simply determines if the new partial pressure of a constituent at a given layer exceeds its saturation pressure for that layer's temperature. If condensation has occurred new mole fractions and temperature are computed. At high altitudes $(P<6.3$ bars $)$, the measured T-P profile (Lindal 1992) is used, and agreement is forced at 6.3 bars. For a more thorough treatment see, e.g., de Pater and Massie (1985) and Atreya and Romani (1985).

\subsection{Radiative Transfer Model}

The radiative transfer model calculates the observed brightness temperature utilizing the computed constituent abundance and temperature profiles from the thermochemical model. For a plane-parallel atmosphere in local thermodynamic equilibrium at a given angle relative to the normal $(\theta$, the "look angle"), neglecting scattering, the emergent flux can be written as

$$
B_{v}(T, \mu)=\int_{0}^{\infty} B_{v}(T(\tau)) E^{-\pi / \mu} \frac{d \tau}{\mu},
$$

where $B_{l}(T)$ is Planck's radiation law, $\mu=\cos \theta$ is the cosine of the look angle (see de Pater and Massie 1985), and $\tau$ is the optical depth.

In general, the disk averaged brightness temperature of a planet, $T_{1}$, is derived from averaging the brightness temperature distribution over the disk of projected area $A$,

$$
B_{v}\left(T_{\mathrm{D}}\right)=\frac{1}{A} \int_{0}^{2 \pi} \int_{0}^{R_{\mathrm{p}}^{(t, k)}} B_{v}(T, \eta, \psi) \eta d \eta d \psi
$$

where $\eta$ is the radial distance from the center of the disk and $\psi$ is the azimuthal angle. $R_{\mathrm{p}}(\psi)$ is the radius of the planet for a given pressure level. If $\eta=f(\mu, \psi)$ and $B_{v}(T$, $\mu, \psi)$ is independent of $\psi$, this can be rewritten as

$$
B_{\imath}\left(T_{\mathrm{D}}\right)=\int_{0}^{1} B_{l}(T, \mu) \mathbb{A}(\mu) d \mu
$$

where

$$
\mathscr{A}(\mu)=-\frac{1}{A} \int_{0}^{2 \pi} f(\mu, \psi) \frac{\partial f}{\partial \mu} d \psi
$$

$f(\mu=1, \psi)=0$, and $f(\mu=0, \psi)=R_{p}(\psi) . \mathscr{A}(\mu)$ is called the look angle weighting function. For a spherical planet, $\mathscr{A}(\mu)=2 \mu$ and the disk averaged brightness temperature in this case can then be further simplified and written in terms of the exponential integral,

$$
\begin{aligned}
B_{v}\left(T_{\mathrm{D}}\right) & =2 \int_{0}^{\infty} B_{v}(T(\tau)) E_{2}(\tau) d \tau \\
& =-2 \int_{0}^{\infty} B_{l}(T(\tau)) d E_{3}(\tau)
\end{aligned}
$$

where

$$
E_{n}(\tau)=\int_{1}^{\infty} \frac{1}{y^{n}} e^{\cdot y} d y
$$

and $y=1 / \mu$.

If the look angle weighting function is fit to an $\mathrm{Nth}$ order polynomial such that

$$
\mathscr{A}(\mu)=\sum_{n=1}^{N} a_{n} \mu^{n}
$$

then the disk averaged brightness temperature for an oblate spheroid can be written as

$$
\begin{aligned}
B_{r}\left(T_{\mathrm{D}}\right) & =\sum_{n-1}^{N} a_{n} \int_{0}^{\infty} B_{r}(T(\tau)) E_{n+1}(\tau) d \tau \\
& =-\sum_{n=1}^{N} a_{n} \int_{0}^{\infty} B_{n}(T(\tau)) d E_{n+2}(\tau)
\end{aligned}
$$

To a high degree, Neptune can be considered a spherical planet and the spherical approximation may be used (de Pater and Massie 1985). However, for other outer planets. most notably Saturn, sphericity cannot necessarily be assumed and the look angle weighting function concept may be useful for numerically simplifying their study (DeBoer 1995).

\section{OPACITY OF THE ATMOSPHERIC CONSTITUENTS}

Now that the model has been developed, determination of the opacity of the atmospheric constituents is necessary to complete the formulation. A table listing solar abundances is instructive in determining the molecules of impor- 
TABLE I

Solar Abundances of Planetary Components (Mole Fraction)

\begin{tabular}{lcc}
\hline & Cameron (1982) & Anders-Grevasse (1989) \\
\hline $\mathrm{H}_{2}$ & $8.788 \times 10^{1}$ & $8.346 \times 10^{1}$ \\
$\mathrm{Hc}$ & $1.186 \times 10^{1}$ & $1.623 \times 10^{1}$ \\
$\mathrm{H}_{2} \mathrm{O}$ & $1.213 \times 10^{3}$ & $1.424 \times 10^{-3}$ \\
$\mathrm{CH}_{4}$ & $7.338 \times 10^{4}$ & $6.043 \times 10^{+}$ \\
$\mathrm{NH}_{3}$ & $1.529 \times 10^{+}$ & $1.873 \times 10^{+}$ \\
$\mathrm{H}_{3} \mathrm{~S}$ & $3.304 \times 10^{5}$ & $3.081 \times 10^{5}$ \\
$\mathrm{PH}_{3}$ & $4.297 \times 10^{7}$ & $6.222 \times 10^{7}$ \\
\hline
\end{tabular}

tance (see Table I). We will briefly examine the sources of opacity, similar to previous studies (de Pater and Mitchell 1993).

\section{1. $H, S$}

In previous models that have investigated the spectra of the outer planets, the opacity of hydrogen sulfide $\left(\mathrm{H}_{2} \mathrm{~S}\right)$ has been one of the most poorly constrained parameters and is often neglected. With the absorption formalism developed in DeBoer and Steffes (1994) it is possible to more accurately determine the effects of this molecule. Hydrogen sulfide was found to be more opaque than the Van Vleck-Weiskopf models predicted. by at least a factor of two in the wings. The radiative effects of this molecule are therefore more pronounced than previously believed.

\section{2. $\mathrm{NH}_{3}$}

Ammonia $\left(\mathrm{NH}_{3}\right)$ is a critical absorber at centimeter wavelengths due to a series of strong inversion lines near $24 \mathrm{GHz}$. The first accurate formalism to model ammonia's absorption under conditions suitable for the outer planets (Berge and Gulkis 1976) was based on a modified BenReuven lineshape. More recently Spilker (1990) and Joiner et al. (1989) have more thoroughly measured the opacity of ammonia under jovian conditions at centimeter and millimeter wavelengths and newer, more accurate formalisms have been developed.

Spilker's version, though somewhat complex, is very accurate at centimeter wavelengths and is used for frequencies below $40 \mathrm{GH} \%$ as suggested. This formalism was also extrapolated below the lowest temperature measurement at $190 \mathrm{~K}$. Above $40 \mathrm{GHz}$ this formalism can exhibit anomalous behavior (as well as in instances when the $\mathrm{NH}_{3}$ mole fraction is very small) and therefore the formalism developed in Joiner and Steffes (1991) is used in these cases. Although the two formalisms agree very well at $40 \mathrm{GHz}$. a linear transitional period at about $40 \mathrm{GHz}$ is used to avoid any discontinuity in the absorption.

\section{3. $\mathrm{H}_{2} \mathrm{O}$}

Water vapor also strongly affects microwave emissions and is undoubtedly the most thoroughly studied molecule due to its important role on the earth. The broadening parameters of water vapor in a hydrogen/helium atmosphere have also been recently measured near the 183and $380-\mathrm{GHz}$ transitions (Dutta et al. 1993) and been incorporated into a newer formalism for water absorption under jovian conditions. The formalism of this work is based on a terrestrial model (Waters 1976, Ulaby et al. 1981) with a correction term for jovian atmospheres (Goodman 1969), and is roughly a factor of two less than Goodman's at higher frequencies $(>20 \mathrm{GHz})$ and varies from being 10 to $100 \%$ less at lower frequencies (DeBoer 1995).

\section{4. $\mathrm{PH}_{3}$}

Phosphine has been identified on Jupiter and Saturn (Weisstein and Serabyn 1994a,b) and it is therefore included in this model of the outer planets. No laboratory measurements of the microwave or millimeter-wave line parameters of $\mathrm{PH}_{3}$ have been made and therefore its lineshape is purely speculative. By including a range of parameters, however, its potential effects on the atmosphere may be investigated. Phosphine has a strong rotational line at $267 \mathrm{GHz}$ and a rich rotational spectrum above $500 \mathrm{GHz}$ as well as a series of weak inversions lines in the centimeter wavelengths. A Van Vleck-Weiskopf lineshape is assumed with a range of reasonable line broadening parameters and the same self-broadening temperature dependence as $\mathrm{NH}_{3}$ (given its structural similarity to $\mathrm{PH}_{3}$ ). Figure 1 shows the

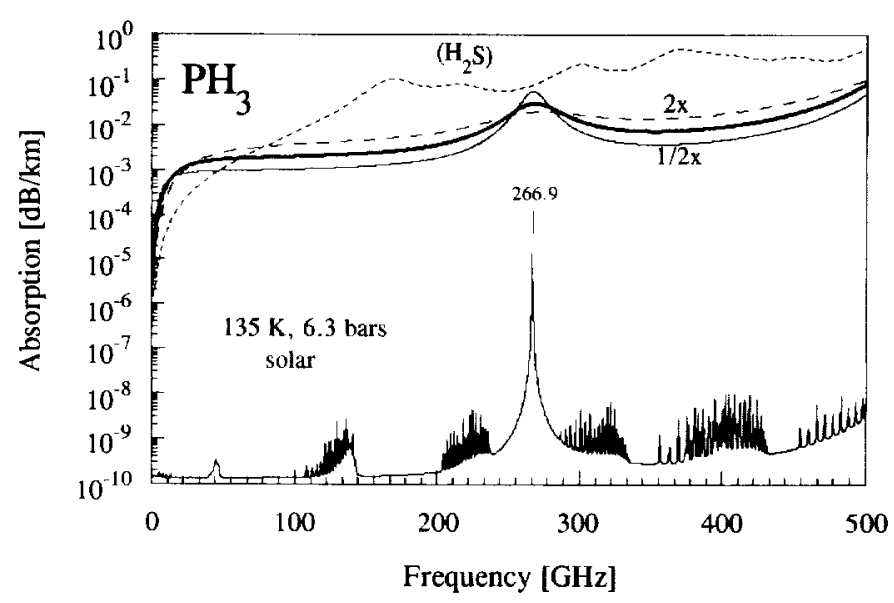

FIG. 1. The microwave and millimeter-wave spectrum of phosphine. For the middle set of spectra, the heavy line is the spectrum with the nominal values for the line broadening parameters used in the text, while the dashed line doubles these parameters and the thin solid line halves them. The lower curve shows the opacity at microbars of pressure to exhibit the individual transition structure. The short-dashed upper curve is the absorption spectrum of $\mathrm{H}_{2} \mathrm{~S}$ with the same mixing ratio as for phospine. 
strong $267-\mathrm{GHz}$ line as well as an absorption spectrum computed for conditions encountered on the outer planets with different line broadening parameters. Three hundred lines are used from the Poynter and Pickett (1985) catalog. including all lines below $1840 \mathrm{GHz}$. Note also in this figure that $\mathrm{H}_{2} \mathrm{~S}$ absorption (with the same mixing ratio as phosphine) is shown.

\subsection{Absorption by Other Constituents}

$\mathrm{CO}$ has four rotational transitions in the millimeter wavelengths and has been identified on the outer planets (e.g., Marten et al. 1993). Like phospine, no laboratory measurements of this molecule under appropriate conditions and frequencies exist, and therefore a range of parameter values will be investigated. The dominant effects from the inclusion of this molecule will be seen to be in the near vicinity of the linecenters and are therefore relatively insensitive to the exact form of the lineshape. A Van Vleck-Weiskopf lineshape is assumed in this model and a total of 26 lines are used, with the highest frequency being $2984 \mathrm{GHz}$.

Under collision, molecular hydrogen $\left(\mathrm{H}_{2}\right)$ exhibits a transient dipole moment which can interact with radiation. Joiner and Steffes (1991) fit an expression similar to Goodman's (1969) to a quantum mechanical model developed by Borysow et al. (1985), which included methane, and is the formalism used for $\mathrm{H}_{2}$ opacity in this work. Absorption by methane itself is negligible in the microwave region and will be neglected (Jenkins and Steffes 1988).

The effects of cloud absorption on the brightness temperature spectrum are considered. The complex refractive index is a key parameter in the determination of the effects of clouds on the brightness temperature spectrum. Unfortunately, there are no microwave data on the refractive index of the liquid or ice phases of the constituents discussed above, with the exception of water. In this work, absorption due to liquid water clouds is included, where the refractive index is computed following King and Smith (1981). The effects are similar to previous studies (de Pater and Mitchell 1993). Scattering at these wavelengths is assumed negligible, as has been shown by others (e.g., de Pater and Mitchell 1993).

The infrared properties of $\mathrm{NH}_{3}$-ice have been measured by Sill et al. (1980). The lowest frequency measured was $50 \mathrm{~cm}^{-1}(1500 \mathrm{GHz})$ with a value of $n^{\prime \prime}=0.016$. The real part has a value of $n^{\prime} \approx 1.4$ at $4 \mu \mathrm{m}$. The complex refractivity of $\mathrm{H}_{2} \mathrm{~S}$-ice has also been measured down to $21 \mathrm{~cm}^{-1}$. where $n^{\prime}=1.15$ and $n^{\prime \prime}=0$ (Pearl, Sill and Ferraro, personal communication). For $\mathrm{H}_{2} \mathrm{~S}$ liquid $n^{\prime}=1.37$ while $n_{\mathrm{NH}_{4} \mathrm{SH}}^{\prime}=$ $1.74(\mathrm{CRC}, 1981)$ and $n_{\mathrm{CH}_{4}}^{\prime}=1.309$ (Baines and Hammel 1994).

\section{OBSERVATIONAL CONSTRAINTS}

There are a number of observational results that can be used in order to constrain which of the models for Neptune may be valid. These include radio occultation results, which yield absorptivity and temperature-pressure profiles, and radio-emission measurements by large radio telescopes. Once constraints on the emission and absorption have been set, modeling will attempt to best fit the data using the model developed in the previous chapter, by selecting different constituent distributions. Of course, the solution obtained is not unique; however, by utilizing all of the available data, realistic constraints on the structure can be set. In this section, the observational constraints and their results will be introduced and discussed.

\subsection{Radio Occultation}

The first constraint is to match the physical temperature measured at the deepest point by the Voyager radio occultation experiment. This is done in the thermochemical model by starting deep within the atmosphere and stepping out, assuming a wet adiabatic lapse rate. Above the deepest measured point the actual measured lapse rate is used. For Neptune, the deepest point probed was at $S$ band $(13 \mathrm{~cm})$, and nominally reached a pressure of 6.27 bars and a temperature of $135 \mathrm{~K}$ (Lindal 1992), with an uncertainty of roughly 1 bar of pressure and $5 \mathrm{~K}$, and maximum values of 7.7 bars and $160 \mathrm{~K}$.

Absorption was also measured on the Voyager link. Lindal attributed the absorption to gaseous ammonia and gives a nominal ammonia mole fraction of $500 \pm 150 \mathrm{ppbv}$. Using Berge and Gulkis (1976), this corresponds to $8.2 \times$ $10^{-4} \pm 2.4 \times 10^{-4} \mathrm{~dB} / \mathrm{km}$ at the $\mathrm{S}$ band. However DeBoer and Steffes (1994) point out that $\mathrm{H}_{2} \mathrm{~S}$ could also account for this absorption. Phosphine could also account for some amount of the measured opacity, as will be seen.

\subsection{Radio-Emission}

Another important set of constraints consists of the radio-emission observations from earth-based radio telescopes. Reliable microwave measurements of Neptune began in the late 1960s and early 1970s. Due to Neptune's extreme distance, however, single dishes at these frequencies cannot resolve the planet from the background. The data therefore are subject to the possibility of background contamination from microwave emitters near the galactic center, as evidenced by the large spread of data with nonoverlapping error bars in Fig. 2.

Multiple dishes used as an interferometer can resolve Neptune from the background and this work therefore emphasizes the data from interferometers (the filled data points in the subsequent figures showing Neptune's measured spectrum) such as the Very Large Array (VLA). 
Even VLA data need be subject to scrutiny, especially at 20 $\mathrm{cm}$ with the rather wide spread in brightness temperature there. The $20-\mathrm{cm}$ emission measurement with the lowest value is the one that will be emphasized, since it is the most recent, and the intent of that VLA observation was specifically to very accurately determine the $20-\mathrm{cm}$ brightness temperature and therefore a very long integration time was used (de Pater et al. 1991). Another difficultly is the low value of the data point at $22 \mathrm{GHz}(1.3 \mathrm{~cm})$ taken by de Pater and Richmond (1989), which seems inconsistent with the rest of the VLA data set. That value will be assumed to be a lower limit due to measurement difficulties, and efforts will not be made to precisely fit it in the modeling. A complete list of prior observations of Neptune as well as their new VLA observations are given in De Pater and Richmond (1989). In addition, more recent measurements of Neptune have been taken and are listed in Table II.

\subsection{Other Constraints}

Based on work by Massie and Hunten (1982) and Gierasch and Conrath (1987), intermediate (or frozen) hydrogen is assumed. Differences in the ortho-para fraction can result in differences of about $20 \%$ at $3 \mathrm{~mm}$ (de Pater and Mitchell 1993). A helium mole fraction of 0.19 (Conrath et al. 1991) is used throughout the model. Significant amounts of methane are also found on Neptune. From IR studies, Baines and Hammel (1994) have determined a tropospheric mole fraction of $3 \%$ for $\mathrm{CH}_{4}$ below the methane cloud. Other observers have determined methane mole

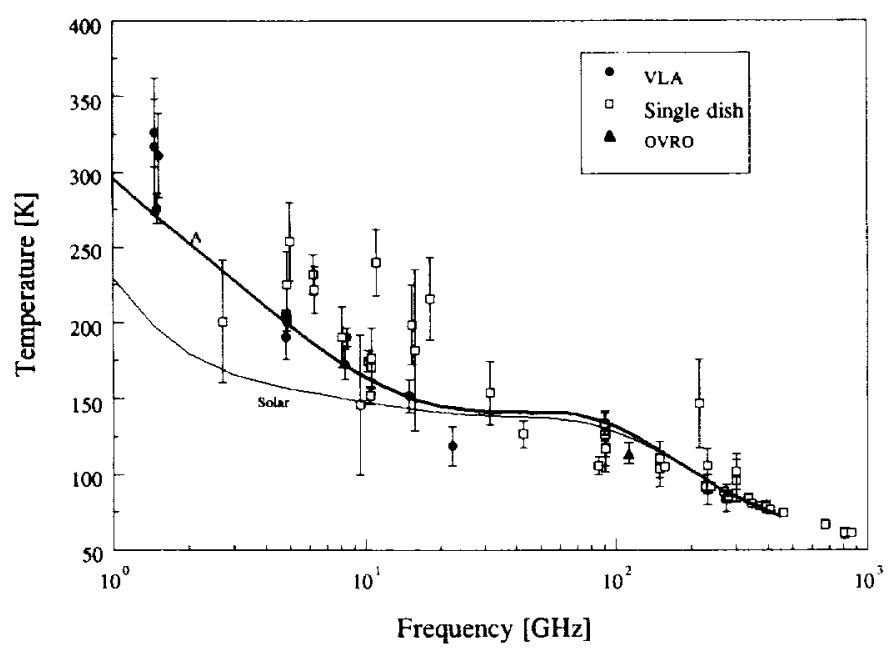

FIG. 2. The measured brightness temperature spectrum of Neptune along with calculations assuming solar abundance values with $3 \%$ methane (labeled "Solar") and distribution A (where A is a solar model in the volatiles' abundances, except that $23 \%$ ammonia and $3 \%$ methane are assumed; see text).
TABLE II

Neptune Observations

\begin{tabular}{lcc}
\hline \multicolumn{1}{c}{ Reference } & $\nu(\mathrm{GHz})$ & $T(\mathrm{~K})$ \\
\hline Romani et al. $(1989)$ & 1.50 & $318 \pm 16$ \\
De Pater et al. (1991) & 1.49 & $276.4 \pm 10$ \\
Muhleman and Berge (1991) & 8.45 & $191.2 \pm 6$ \\
Griffin and Orton (1993) & 112.8 & $114 \pm 7$ \\
& 90 & $133.9 \pm 7.4$ \\
& 150 & $110.3 \pm 3.6$ \\
& 156 & $105.3 \pm 2.3$ \\
& 227 & $91.9 \pm 3.5$ \\
& 237 & $91.9 \pm 1.8$ \\
& 269 & $88.3 \pm 1.8$ \\
& 279 & $85.4 \pm 3.9$ \\
& 337 & $84.1 \pm 3.0$ \\
& 347 & $80.6 \pm 1.7$ \\
& 374 & $79.5 \pm 2.5$ \\
& 392 & $78.7 \pm 3.8$ \\
Greve et al. (1994) & 410 & $76.8 \pm 1.8$ \\
& 460 & $74.9 \pm 1.9$ \\
& 674 & $66.8 \pm 3.1$ \\
& 808 & $61.0 \pm 3.5$ \\
& 858 & $61.5 \pm 2.7$ \\
& 4.84 & $191 \pm 15$ \\
& 8.3 & $173 \pm 10$ \\
& 43 & $127 \pm 9$ \\
\hline
\end{tabular}

fractions of 2-4\% (see, e.g., Orton et al. 1986, de Pater and Massie 1985, Lindal 1992). This work assumes $3 \% \mathrm{CH}_{4}$.

\section{RESULTS}

The modeling will be broken into two different distribution types, ammonia predominant atmospheres and hydrogen sulfide predominant atmospheres, which are discussed below.

\subsection{Ammonia Predominant Atmospheres}

If one assumes a strictly solar abundance atmosphere (see Table I) ${ }^{1}$, the result is an ammonia predominant atmosphere. The ammonia will deplete the hydrogen sulfide in the putative ammonium hydrosulfide cloud located near the 12-bar pressure level. In this case it is apparent that discrepancies exist at low frequencies (below $20 \mathrm{GHz}$ ) where the model spectrum is far too cold (see Fig. 2). This is due to $\mathrm{NH}_{3}$ present in the upper atmosphere. Furthermore, the Owens Valley data point at $112 \mathrm{GHz}$ suggests that a greater source of opacity is needed at the altitude probed by this frequency, as does the Greve et al. (1994) point at $43 \mathrm{GHz}$. Obviously some $\mathrm{NH}_{3}$ (the dominant microwave absorber in this distribution) needs to be removed

\footnotetext{
' For consistency with other works, the Cameron abundances, as summarized in Atreya 1986, will be used as the reference.
} 


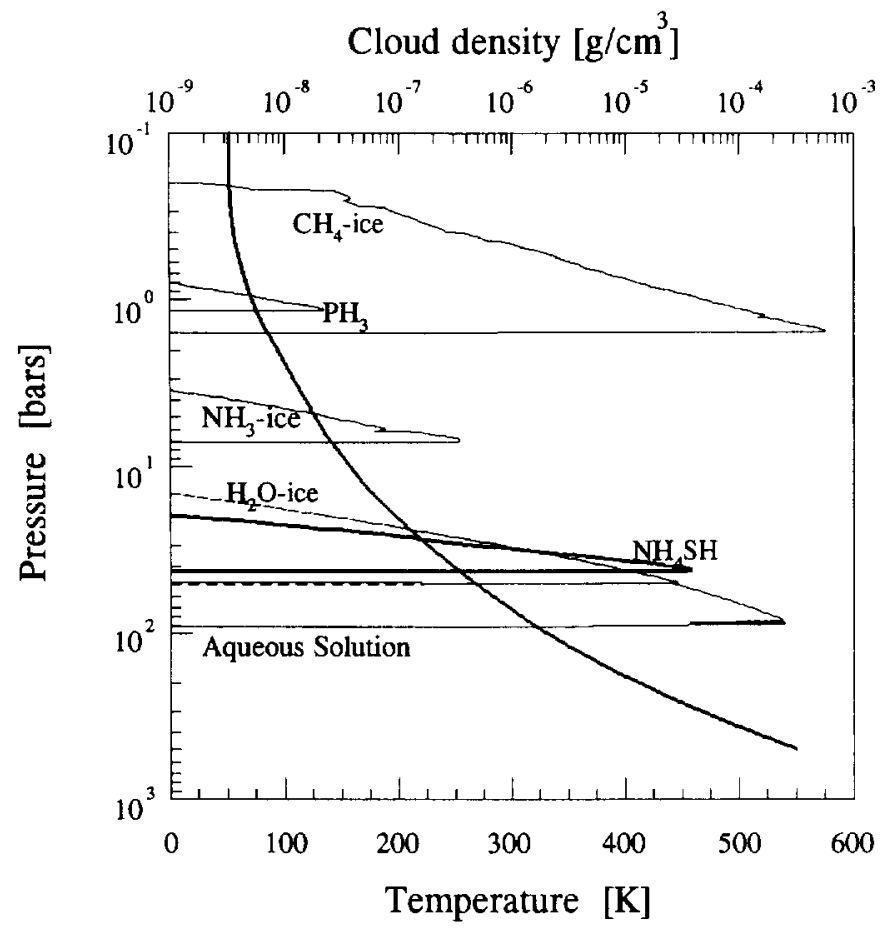

FIG. 3. Cloud structure and temperature profile of distribution A.

and a millimeter absorber added, relative to solar abundances.

There are essentially three ways to deplete $\mathrm{NH}_{3}$ in the upper reaches of the troposphere (upper here meaning $P \leq 20$ bars): (1) start out with less ammonia in the deep atmosphere, (2) add $\mathrm{H}_{2} \mathrm{~S}$ to remove $\mathrm{NH}_{3}$ via the $\mathrm{NH}_{4} \mathrm{SH}$ cloud, or (3) add more water to remove it via the aqueous solution cloud. The very warm data at $20 \mathrm{~cm}(1.5 \mathrm{GHz})$ and the slope of the spectrum between 20 and $1 \mathrm{~cm}$ indicate that subsolar quantities of ammonia are present below the putative $\mathrm{NH}_{4} \mathrm{SH}$ cloud and hence options (1) and/or (3) are valid. The apparent low abundance of ammonia does not necessarily imply that nitrogen is globally depleted since other reservoirs for it may exist, primarily $\mathrm{N}_{2}$, as suggested by Marten et al. (1993).

Decreasing the amount of ammonia in the deep atmosphere from that of a solar abundance (but keeping $X_{\mathrm{H}_{2} \mathrm{~S}}<X_{\mathrm{NH}_{3}}$ ) allows very good fits to the VLA data below $20 \mathrm{GHz}$; however, the spectrum is too bright for frequencies greater than $40 \mathrm{GHz}$ as evidenced by the Greve et al. (1994) and the Owens Valley Radio Observatory (OVRO) data (Muhlemann and Berge 1991). In Fig. 2, curve A shows one of the best fits when assuming that $\mathrm{NH}_{3}$ is the dominant absorber in an upper troposphere that contains solar $\mathrm{H}_{2} \mathrm{~S}, 23 \%$ solar $\mathrm{NH}_{3}$, solar $\mathrm{H}_{2} \mathrm{O}, 40 \times$ solar $\mathrm{CH}_{4}$, and solar $\mathrm{PH}_{3}$ (distribution $\mathrm{A}$ ). Figure 3 shows the T-P profile and cloud structure of distribution $\mathrm{A}$, and Fig. 4 shows the weighting function at several frequencies. However, this distribution results in an S-band $(13 \mathrm{~cm})$ absorptivity at the 6.3-bar level of $2.31 \times 10^{-3} \mathrm{~dB} / \mathrm{km}$, which is roughly a factor of 3 greater than the absorption measured by Voyager and more than a factor of 2 greater than the upper limit given for the Voyager results in Lindal (1992).

Another major problem with distributions similar to that of $\mathrm{A}$ is that they are too warm at millimeter wavelengths. Adding even large amounts of $\mathrm{NH}_{3}$ to cool the millimeterwave portion of the synthetic spectra to fit the measured data is not sufficient, and this drastically cools the centimeter portion to be inconsistent with the VLA data, as seen in the distribution assuming solar abundances. An absorber is needed in the upper troposphere either in addition to $\mathrm{NH}_{3}$ or in lieu of it. Some candidates for increasing the millimeter-wave opacity in addition to ammonia in the troposphere are phosphine, supersaturations of hydrogen sulfide, carbon monoxide, and cloud particulates. Adding these constituents will also be seen to affect the centimeter wavelength absorptivity.

5.1.1. Phosphine. Increasing the phosphine abundance is an effective way to cool the spectrum at frequencies greater than $40 \mathrm{GHz}$ not only because of its strong set of millimeter wavelength lines but also because it is one of

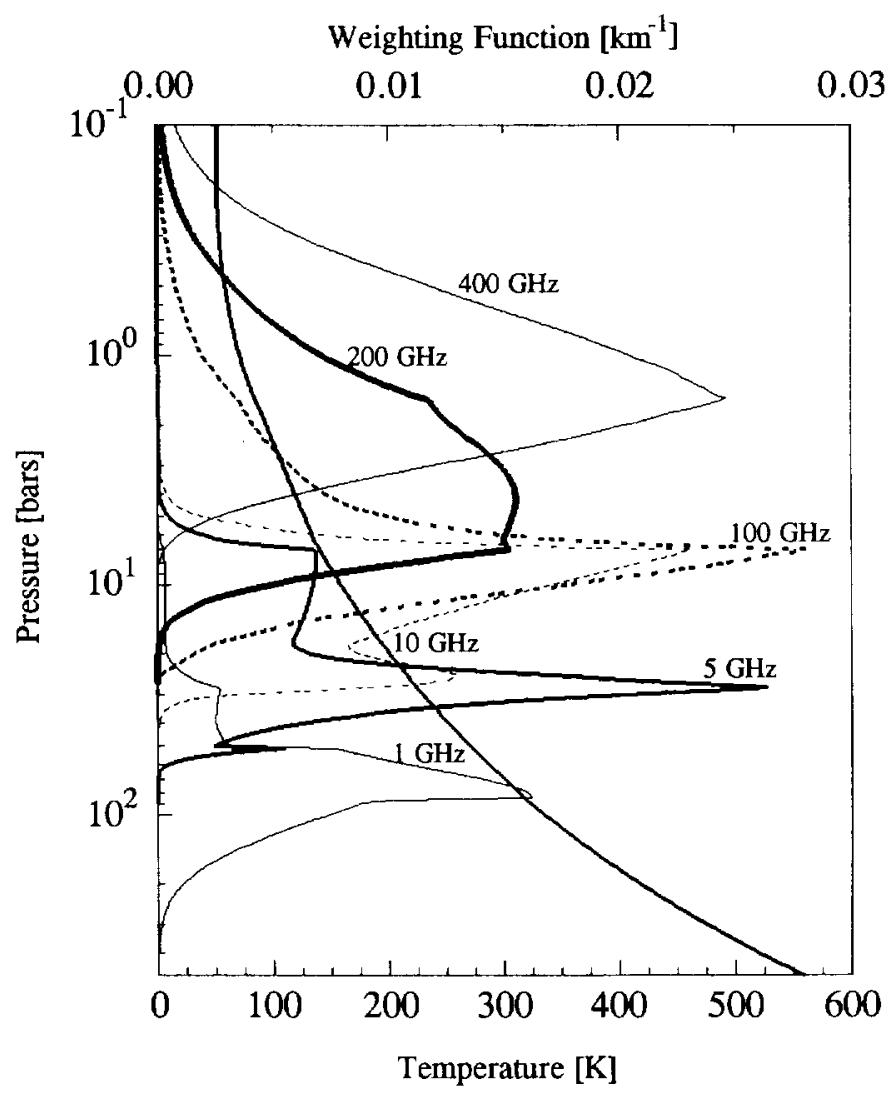

FIG. 4. Weighting functions of distribution $A$, along with the temperature-pressure profile. 


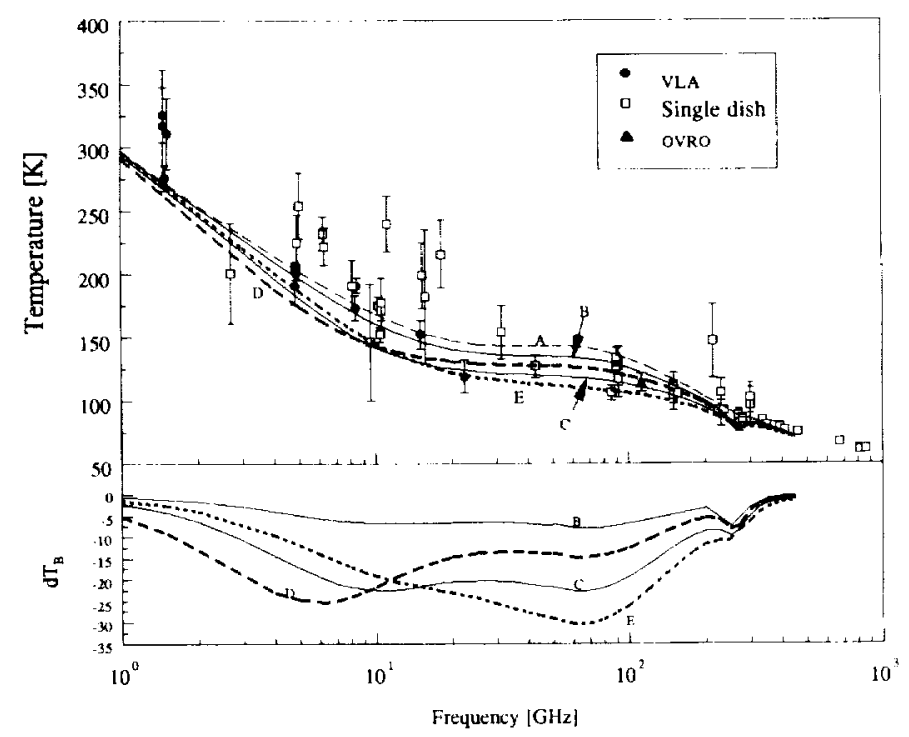

FIG. 5. Measured and synthetic spectra of Neptune with varying amounts of phosphine. The capital letters correspond to spectra discussed in the text. $\mathrm{B}$ and $\mathrm{C}$ are the same as $\mathrm{A}$ except that $\mathrm{PH}_{3}$ is enhanced by 20 and 100 times the solar value, respectively, while $\mathrm{D}$ and $\mathrm{E}$ examine the effects of halving and doubling the line broadening parameters of distribution $C$. The bottom shows the difference of each synthetic spectra from that of distribution $A$.

the last volatiles to condense out, therefore resulting in fairly long path lengths through it, even at the higher frequencies. Furthermore, it has been detected on Jupiter in roughly solar amounts (Weisstein and Serabyn 1994b) and on Saturn at about $10 \times$ solar amounts (Weisstein and Serabyn 1994a) and is therefore known to be present on the outer planets.

Curve B in Fig. 5 shows the spectrum of the same distribution as $A$ but with phosphine enhanced $20 \times$ its solar value (distribution $B$ ) and has an absorption coefficient at 6.3 bars at the $S$ band calculated to be $2.82 \times 10^{-3} \mathrm{~dB} /$ $\mathrm{km}$, about 3.5 times that measured by Voyager. Curve $\mathrm{C}$ in Fig. 5 has a $\mathrm{PH}_{3}$ enhancement of 100 (distribution $\mathrm{C}$ ) with an S-band absorptivity of $4.99 \times 10^{-3} \mathrm{~dB} / \mathrm{km}$. This does cool down the spectrum at millimeter wavelengths as well as at centimeter wavelengths. Keep in mind, however, that the opacity of phosphine under these conditions has never been measured. Curve D in Fig. 5 shows what happens if the line broadening parameters for $\mathrm{PH}_{3}$ in distribution $\mathrm{C}\left(100 \times\right.$ solar $\left.\mathrm{PH}_{3}\right)$ are cut in half, while curve $\mathrm{E}$ shows when they are doubled. Curves $D$ and $E$ have S-band absorptivities of $7.54 \times 10^{-3}$ and $3.74 \times 10^{-3}$ $\mathrm{dB} / \mathrm{km}$, respectively.

It is quite interesting to note that the spectra calculated with distributions $D$ and E cross just above $10 \mathrm{GHz}$. This is due to the effects of the extremely weak inversion lines at low frequency. When the line broadening parameters are large, the opacity from the low-frequency transitions gets diluted to the point that they essentially contribute no net opacity and hence the spectrum warms up. For the stronger lines at higher frequencies, broadening has the opposite effect of cooling the synthetic spectra. The converse of this is true when the line broadening parameters are narrowed.

By including approximately 10-20 times the solar amount of $\mathrm{PH}_{3}$, therefore, it is seen that the reliable radioemission measurements may be fit, depending on the choice of lineshape and its parameter values. Ammonia predominant atmospheres with supersolar amounts of phosphine suffer, however, by significantly exceeding the atmospheric opacity measured by Voyager radio occultation. Clearly, laboratory measurements are needed of the opacity due to phosphine to help resolve the effects of $\mathrm{PH}_{3}$ (Fig. 1 indicates that the opacity of phosphine should easily be measurable in the laboratory); but the fact remains that the opacity at $S$ band at the 6.3-bar level of such an atmosphere would be excessive.

5.1.2. $\mathrm{H}_{2} \mathrm{~S}$ supersaturation. Another possibility in matching the observed spectrum is through allowing supersaturation to occur. Bezard et al (1983) and de Pater et al. (1991) have noted that the formation of $\mathrm{NH}_{4} \mathrm{SH}$ (the coupling agent between $\mathrm{NH}_{3}$ and $\mathrm{H}_{2} \mathrm{~S}$ ) may require significant reaction surfaces (i.e., many sticky aerosols upon which to form) and supersaturations of $\mathrm{H}_{2} \mathrm{~S}$ and $\mathrm{NH}_{3}$ with respect to $\mathrm{NH}_{4} \mathrm{SH}$ may be likely. Accordingly, the radiative transfer code has been modified to account for this. Allowing $\mathrm{H}_{2} \mathrm{~S}$ to be supersaturated with respect to ammonia in the ammonium hydrosulfide cloud achieves essentially the same effect as adding phosphine. Spectra resulting from supersaturations of 1 and 5 ppmv correspond roughly to curves $\mathrm{B}$ and $\mathrm{D}$, respectively, in Fig. 5, with the exception that $\mathrm{H}_{2} \mathrm{~S}$ supersaturations do not cool the millimeter-wave spectrum as effectively as spectra obtained by adding $\mathrm{PH}_{3}$. Both 1 and 5 ppmv have absorptivities at 6.3 bars of about 3 times that measured by Voyager.

5.1.3. Carbon monoxide. $\mathrm{CO}$ has been detected in emission on Neptune (Marten et al. 1991, 1993, Rosenqvist et al. 1992) while Guilloteau et al. (1993) have detected this molecule in absorption, showing that $\mathrm{CO}$ exists in the troposphere as well as in the stratosphere. Guilloteau et al. derive a mole fraction of roughly $10^{-6}$ and find the likely source of $\mathrm{CO}$ to be from the interior of the planet, rather than from cometary infall or some other external source. Neptune's strong internal heat source and the consequent convective upwelling provide a mechanism for the transport of this molecule from its interior (Lodders and Fegley 1994). The results of including 1 ppmv of $\mathrm{CO}$ throughout the troposphere in the model are seen in Fig. 6, which is seen to be identical to distribution $A$ except near the $\mathrm{CO}$ lines where rather deep absorption lines are seen. The presence of $\mathrm{CO}$ is consistent with the data near the 115 - 


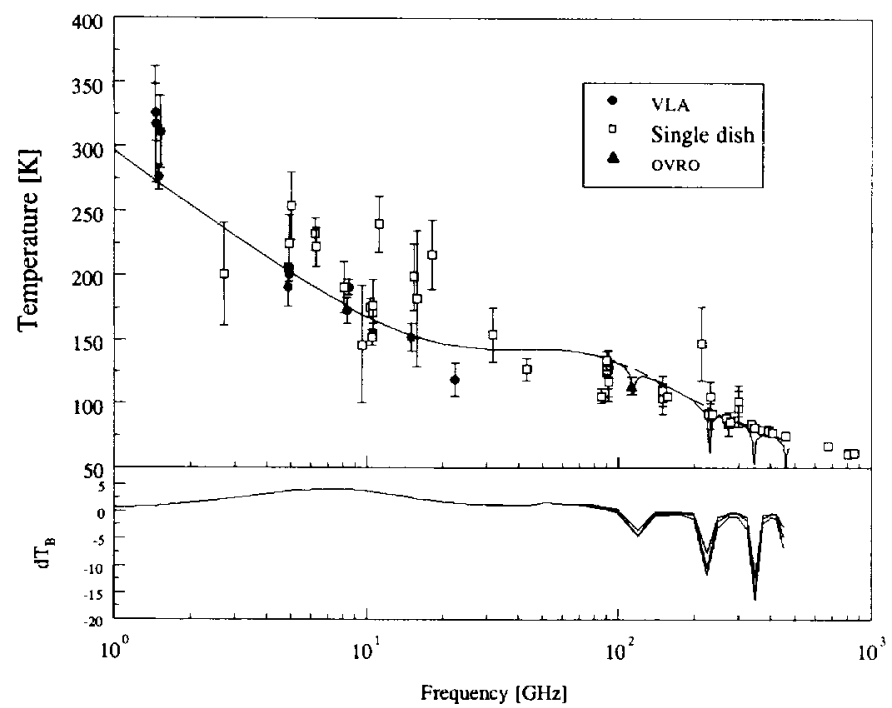

FIG. 6. Measured and synthetic spectra of Neptune with the effects of $1 \mathrm{ppmv} \mathrm{CO}$ throughout the troposphere. The bottom part of the figure shows the differential between distribution $A$ and atmospheres containing $\mathrm{CO}$, including the effects of halving and doubling the line broadening parameters. It is seen that the spectra are fairly insensitive to the line broadening parameters of $\mathrm{CO}$, except near the linecenters.

GHz absorption line but not the single dish data near the higher absorption lines. The lack of consistency at high frequency could stem from errors in the observations as well as in the model, which is optimized for centimeter and millimeter wavelengths. It is seen, however, that the effects of $\mathrm{CO}$ are confined to relatively narrow regions about the linecenter and the presence of $\mathrm{CO}$ cannot be used to shape the entire synthetic microwave spectra effectively.

5.1.4. Conclusion. By using the above four atmospheric constituents $\left(\mathrm{NH}_{3}, \mathrm{H}_{2} \mathrm{~S}, \mathrm{PH}_{3}\right.$, and $\left.\mathrm{CO}\right)$ one can fit the measured emission spectrum relatively well at all frequencies; however, the resulting $13-\mathrm{cm}$ opacity still exceeds that measured at 6.3 bars in the ammonia dominant atmospheres. This situation worsens as one adds constituents to better fit the millimeter wavelength data (except $\mathrm{CO}$ ), as has been shown. Decreasing the amount of ammonia to allow the S-band absorptivity to be matched destroys the spectral fit. Adding another absorber (e.g., phosphine) to regain a good fit to the measured radio-emission spectrum then destroys the match to the S-band absorptivity. It therefore appears that there is no ammonia predominant atmospheric distribution consistent with thermochemical modeling which can provide fits to the measured radio emission as well as to the measured absorptivity. The following section will examine $\mathrm{H}_{2} \mathrm{~S}$ predominant atmospheres to ascertain whether they can resolve the dilemma caused by the radio-emission and absorptivity measurements being apparently at odds.

\subsection{Hydrogen Sulfide Predominant Atmospheres}

By assuming that the abundance of $\mathrm{H}_{2} \mathrm{~S}$ is greater than that of $\mathrm{NH}_{3}$ below the putative $\mathrm{NH}_{4} \mathrm{SH}$ cloud, the reliable and consistent constraining data points can be fit with an $\mathrm{H}_{2} \mathrm{~S}$ predominant atmosphere. One such model assumes 40 times solar $\mathrm{H}_{2} \mathrm{~S}, 20 \%$ solar $\mathrm{NH}_{3}$, solar $\mathrm{H}_{2} \mathrm{O}, 3 \% \mathrm{CH}_{4}$, and solar $\mathrm{PH}_{3}$ (distribution $\mathrm{F}$ ). The resulting spectrum from this distribution is shown by curve $F$ in Fig. 7 and has an S-band absorptivity at 6.3 bars of $4.39 \times 10^{-4} \mathrm{~dB} / \mathrm{km}$, which is below the lower limit of the measured absorption. Figure 8 shows the T-P and cloud profiles for distribution F.

Adding more $\mathrm{H}_{2} \mathrm{~S}$ in order to better fit the absorption at 6.3 bars is ineffective since one is already on the $\mathrm{H}_{2} \mathrm{~S}$ saturation curve and any additional $\mathrm{H}_{2} \mathrm{~S}$ condenses out. $\mathrm{H}_{2} \mathrm{~S}$ supersaturations of about $80 \%$ with respect to its ice cloud are needed to match the S-band absorption. Supersaturations that large seem unlikely in the dense environment in which the putative $\mathrm{H}_{2} \mathrm{~S}$ cloud exists, although strong convective upwelling could conceivably account for it. Supersaturations of water-ice in the much less dense upper terrestrial atmosphere typically do not exceed a few percent. The effect of an $80 \% \mathrm{H}_{2} \mathrm{~S}$ supersaturation with respect to its ice-cloud on the brightness temperature spectrum is very similar to the effect of an $\mathrm{NH}_{3}$ supersaturation of $400 \mathrm{ppb}$ with respect to the $\mathrm{NH}_{4} \mathrm{SH}$ cloud, as well as to the effect of $\mathrm{PH}_{3}$ enhancements of about 10 times the solar value, both of which are discussed below. The broad features of the weighting functions are similar to that of

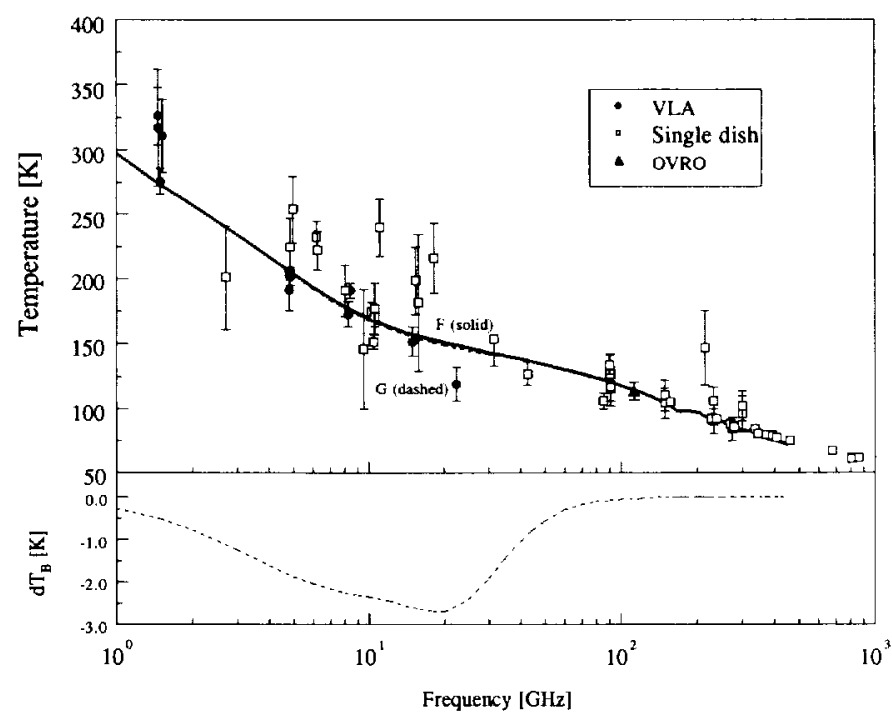

FIG. 7. Measured and synthetic spectra of Neptune with an $\mathrm{H}_{2} \mathrm{~S}$ predominant atmosphere. The capital letters correspond to spectra discussed in the text. Distribution $F$ shows the nominal synthetic spectrum in an $\mathrm{H}_{2} \mathrm{~S}$ atmosphere $\left(X_{\mathrm{H}_{2} \mathrm{~S}}>X_{\mathrm{NH}_{3}}\right)$, while $\mathrm{G}$ has a small $\mathrm{NH}_{3}$ supersaturation $(400 \mathrm{ppb})$. The lower curve shows the difference between distributions $F$ and $G$. 


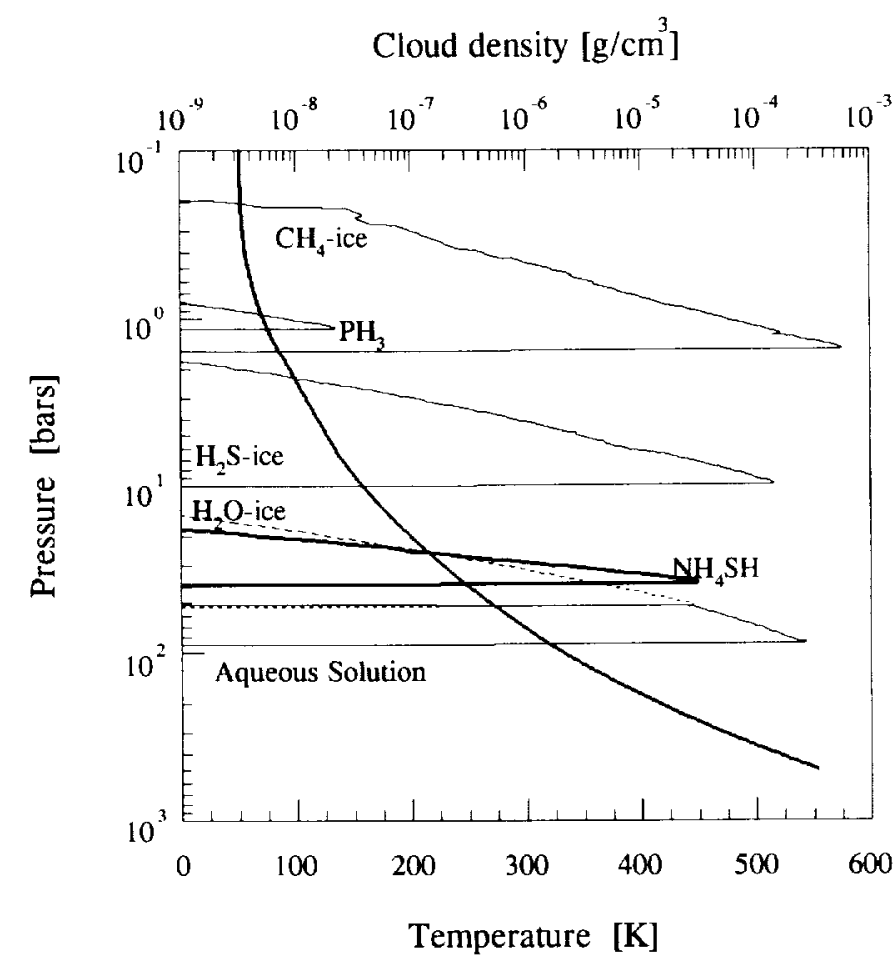

FIG. 8. Temperature-pressure and cloud profiles for distribution F.

Fig. 4. As in the case of the ammonia predominant atmosphere, the effects of the other sources of opacity will be examined individually.

5.2.1. $\mathrm{NH}_{3}$ Supersaturation. Adding ammonia supersaturations with respect to the $\mathrm{NH}_{4} \mathrm{SH}$ cloud has the relatively small effect of increasing the slope of the spectrum at centimeter wavelengths but does allow the 6.3 opacity to be fit arbitrarily well. Curve G in Fig. 7 shows the effect of 400-ppb supersaturation (distribution G), while the bottom graph shows the differential between $F$ and G. Note that Lindal (1992) states that the measured absorption from the S-band radio occultation was due to about 500 $\mathrm{pph}$ of $\mathrm{NH}_{3}$, which accounted for all of the measured absorption. Here, the $400 \mathrm{ppb}$ accounts for about half of the measured opacity due to the fact that this analysis uses the more accurate $\mathrm{NH}_{3}$ absorption formalism (Spilker 1990) which shows ammonia having less opacity than the Berge and Gulkis formalism predicts. At this point (6.3 bar and $135 \mathrm{~K}$ ), both $\mathrm{H}_{2} \mathrm{~S}$ and $\mathrm{NH}_{3}$ are condensing to follow the saturation curve, as stated by Lindal. Supersolar amounts of hydrogen sulfide along with ammonia supersaturation have been previously postulated to explain the Voyager 2 results (de Pater et al 1991); however, the opacity of $\mathrm{H}_{2} \mathrm{~S}$ was not known and the amounts were speculative.

5.2.2. Phosphine and CO. Adding phosphine has roughly the same affect as did $\mathrm{NH}_{3}$ supersaturations; namely, marginal improvement to the fit to the radio-emission spectrum and allowing the S-band opacity measured at the 6.3 bar-level to be fit arbitrarily well. Curves $\mathrm{H}$ and $\mathrm{I}$ in Fig. 9 show distribution F with 10 and 20 times the solar amount of $\mathrm{PH}_{3}$, respectively, and provide very convincing spectral fits. These amounts of phosphine bracket the measured S-band opacity, but both are still within the quoted error bars. An intermediate amount of phosphine can exactly match the quoted opacity. Therefore, phosphine present at approximately 15 times solar along with superabundances of $\mathrm{H}_{2} \mathrm{~S}$ (40 times solar) seem one likely explanation for the measured radio-emission spectrum and S-band absorptivity.

The inclusion of $\mathrm{CO}$ has a similar but smaller effect than in the ammonia predominant atmosphere since there is already additional opacity near the $\mathrm{CO}$ linecenters due to $\mathrm{H}_{2} \mathrm{~S}$ absorption. This makes the troughs in the spectrum at the $\mathrm{CO}$ linecenters more shallow, as evidenced in Fig. 10, which also shows the spectrum with $15 \times \mathrm{PH}_{3}$ and 1 ppmv CO.

5.2.3. Conclusion. $\mathrm{H}_{2} \mathrm{~S}$ predominant atmospheres therefore allow a good fit to the measured emission spectrum, and, by increasing the amount of some other constituent(s), the measured absorptivity can also be fit arbitrarily well. This ability to fit all of the constraints make an $\mathrm{H}_{2} \mathrm{~S}$ dominant atmosphere, with either a supersaturation of ammonia (of about $400 \mathrm{ppb}$ ) or 10-20 times the solar amount of phosphine, a plausible model for the atmosphere of Neptune.

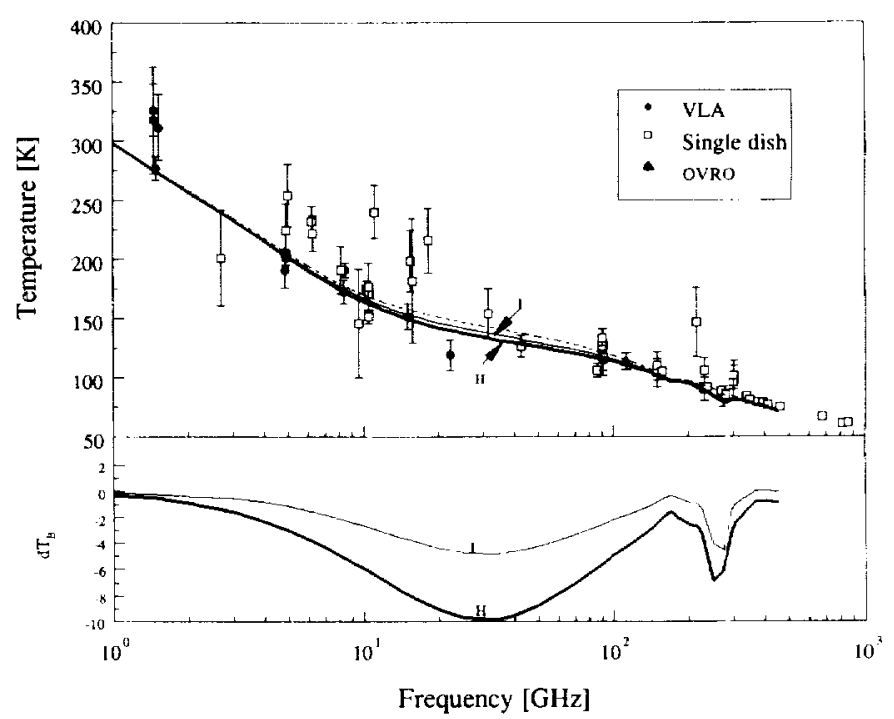

FIG. 9. Measured and synthetic spectra of Neptune assuming distribution $\mathrm{F}$ (dashed line) but with 20 times $(\mathrm{H})$ and 10 times (I) the solar amount of phosphine. The lower curve shows the differential between these enhanced phosphine spectra and distribution $F$. 


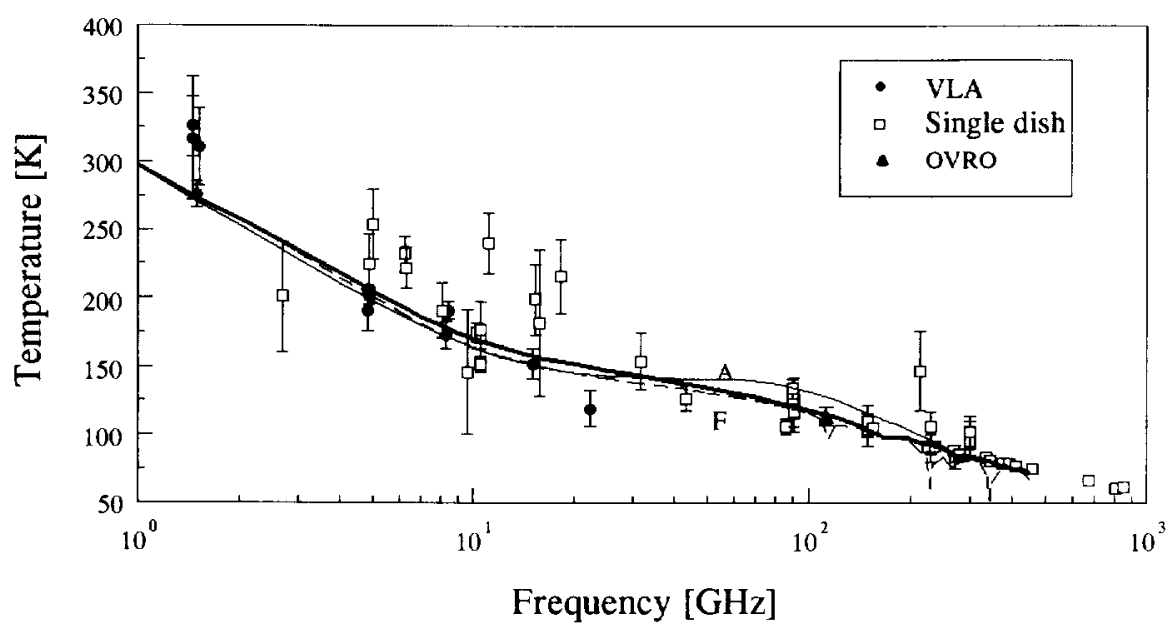

FIG. 10. Comparison of distributions $\mathrm{A}\left(\mathrm{NH}_{3}\right.$ dominant atmosphere: thin line) and $\mathrm{F}\left(\mathrm{H}_{2} \mathrm{~S}\right.$ dominant atmosphere; thick line). Also shown is distribution $\mathrm{F}$ with $15 \times$ solar phosphine and 1 ppmv CO (dashed line).

\section{CONCLUSIONS AND POSSIBILITIES FOR FUTURE STUDIES}

The atmosphere of Neptune has been analyzed assuming it to be either an ammonia predominant or a hydrogen sulfide predominant atmosphere. One conclusion, based on comparison with radio emission, is that the $\mathrm{H}_{2} \mathrm{~S}$ to $\mathrm{NH}_{3}$ ratio is either nearly (but less than) unity (resulting in the ammonia predominant atmosphere) or much greater than unity (a hydrogen sulfide atmosphere). The nominal $\mathrm{NH}_{3}$ atmosphere (distribution $\mathrm{A}$ ) has $\mathrm{H}_{2} \mathrm{~S} / \mathrm{NH}_{3} \approx 0.88$, while the nominal $\mathrm{H}_{2} \mathrm{~S}$ atmosphere (distribution $\mathrm{F}$ ) has $\mathrm{H}_{2} \mathrm{~S} / \mathrm{NH}_{3} \approx 40$. In either case, this ratio is greater than the solar value of 0.2 .

Figure 10 illustrates the difference between the nominal $\mathrm{H}_{2} \mathrm{~S}$ and $\mathrm{NH}_{3}$ models. The abundances of other constituents (primarily $\mathrm{PH}_{3}$ ) can be varied to match the measured spectrum reasonably well regardless of whether $\mathrm{H}_{2} \mathrm{~S}$ or $\mathrm{NH}_{3}$ dominates. However, when including the measured absorptivity at $\mathrm{S}$ band at the 6.3-bar level from Voyager, $\mathrm{NH}_{3}$ predominating models have absorptivities that are too large and only get larger as one better fits the spectrum, whereas $\mathrm{H}_{2} \mathrm{~S}$ models are somewhat too small and approach the measured absorption as one adds other constituents to improve the fit. Figure 11 shows $2.3-\mathrm{GHz}$ absorptivity profiles for some possible abundance distributions discussed in the text. In Fig. 11, distributions A and F clearly bracket the measured opacity at the 6.3-bar level. Distribution $\mathrm{G}$ (which has $400 \mathrm{ppb}$ of $\mathrm{NH}_{3}$ supersaturation) and the enhanced $\mathrm{PH}_{3}$ distribution both match the measured opacity, as well as the measured radio-emission spectrum. It is therefore concluded that the most probable atmospheric distribution, based on the thermochemical modeling and constrained by the measured radio-emission spectrum and measured absorption at the 6.3-bar level, is that of an $\mathrm{H}_{2} \mathrm{~S}$ predominant atmosphere $\left(\mathrm{H}_{2} \mathrm{~S} / \mathrm{NH}_{3} \approx 40\right)$ with either a small supersaturation of $\mathrm{NH}_{3}(400 \mathrm{ppb})$ or $\mathrm{PH}_{3}$ enhanced by approximately a factor of 15 times the solar amount, or some combination of the two.

It is interesting to note that for a given mole fraction and environment, phosphine is more opaque than $\mathrm{H}_{2} \mathrm{~S}$ at

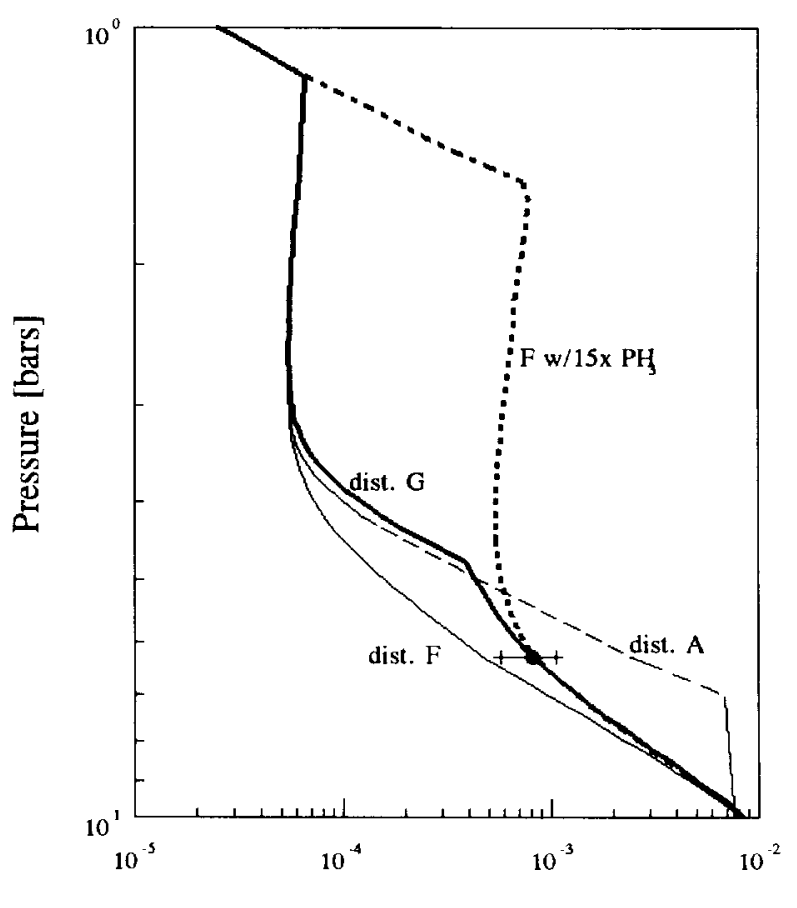

Absorption $[\mathrm{dB} / \mathrm{km}]$

FIG. 11. Calculated absorptivity profiles at $2.3 \mathrm{GHz}$ for Neptune's upper atmosphere along with the Voyager data point. Distribution $\mathrm{G}$ has an $\mathrm{NH}_{3}$ supersaturation of $4(10 \mathrm{ppb}$, while the profile labeled "Fw/15 $\times$ $\mathrm{PH}_{3}$ " has 15 times the solar abundance of $\mathrm{PH}_{3}$. 
centimeter wavelengths, as evidenced in Fig. 1, which also shows $\mathrm{H}_{2} \mathrm{~S}$ absorption with the same mixing ratio as $\mathrm{PH}_{3}$. This figure supports the argument that $\mathrm{PH}_{3}$ is potentially a large source of the opacity even at centimeter wavelengths on the giant planets, a conclusion borne out by the analysis of the previous discussion. It is perhaps the last important constituent of which there are no laboratory data under appropriate conditions.

\section{ACKNOWLEDGMENTS}

The authors thank Imke de Pater and Mark Hofstadter for helpful advice and suggestions. This work was supported by the Planetary Atmospheres Program of the National Acronautics and Space Administration under Grant NAGW-533.

\section{REFERENCES}

Anders, E., And N. Grevesse 1989. Abundances of the elements: Meteoritic and solar. Geochim. Cosmochim. Acta 53, 197-214.

APPLEBY, J. F. 1986. Radiative-convective equilibrium models of Uranus and Neptune. Icarus 65, 383-405.

Appleby, J. F., AND J. S. Hogan 1984. Radiative-convective equilibrium models of Jupiter and Saturn. Icarus 59, 336-366.

Atrey A, S. K. 1986. Atmospheres and Ionospheres of the Outer Planets and Their Satellites. Springer-Verlag, Berlin.

Atreya. S. K., AND P. N. Romani 1985. Photochemistry and clouds of Jupiter. Saturn and Uranus. In Recent Advances in Planetary Meteorology (G. E. Hunt, Ed.). Cambridge Univ. Press, Cambridge.

Baines. K. H., And H. B. Hammel 1994. Gases, hazes and the stratospheric methane abundance in Neptune. Icarus 109, 20-39.

BaINES. K. H., AND W. H. SMITH 1990 . The atmospheric structure and dynamical properties of Neptune derived from ground-based and IUE spectrophotometry. Icarus $\mathbf{8 5 , 6 5 - 1 0 8 .}$

BERGie, G. L., AND S. Gulkis 1976. Earth-based radio observations of Jupiter: Millimeter to meter wavelengths. In Jupiter (T. Gehrels, Ed.) pp. 621-692. Univ. of Arizona Press, Tucson.

Bezard, B., A. Marten, J. P. Balutheal, D. Gaujer, J.-M. Flatid, AND C. CAMY-PEYRET 1983. On the detectability of $\mathrm{H}_{2} \mathrm{~S}$ in Jupiter larus 55, 259-271.

Borysow, J.. L. Trafton, and L. Frommhold 1985. Modeling of pressure-induced far-infrared absorption spectra: Molecular hydrogen pairs. Astrophys. J. 296, 644-654

Cameron, A. G. W. 1982. Elementary and nuclidic abundances in the solar system. In Essays in Nuclear Astrophysics (C. A. Burnes, D. D. Clayton, and D. N. Schramon Eds.), pp. 23-43. Cambridge Univ. Press, Cambridge.

Conrath, B. J.. D. Gautier, G. F. Lindal., R. E. Samilelson, and W. A. SChafFer 1991. The helium abundance of Neptune from Voyager measurements. J. Geophys. Re's. 96, 18,907-18.919.

Conrath, B. J., R. A. Hanel, and R. E. Samuelson 1989. Thermal structure and heat balance of the outer planets. In Origin and Evolution of Planetary and Satellite Atmospheres (S. K. Atreya, J. S. Pollack, and M. S. Matthews, Eds.). pp. 539-563. Univ. of Arizona Press, Tucson.

CRC Press 1981. CRC Handbook of Chemistry and Physics (R. C. Weast, Ed.). CRC Press, Boca Raton, FL.

DeBoer, D. R. 1995. The Microwave Opacity of $\mathrm{H}_{2} \mathrm{~S}$ with Applications to the Tropospheric Verrical Structure of the Jovian Planets. Ph.D. thesis, Georgia Institute of Technology.
DeBoer, D. R., AND P. G. SiffFFs 1994. Laboratory measurements of the microwave properties of $\mathrm{H}_{2} \mathrm{~S}$ under simulated jovian conditions with an application to Neptune. Icarus 109, 352-366.

DE PATER, I., ANi S. T. Massie 1985. Models of the millimeter-centimeter spectra of the giant planets. Icarus 62, 143-171.

DE: PATER, I., AND D. L. MITCHEL.I. 1993. Radio observations of the planets: The importance of laboratory measurements. J. Geophys. Res. 98, $5471-5490$

DE: PATER, I., AND M. Richmond 1989, Neptune's microwave spectrum from $1 \mathrm{~mm}$ to $20 \mathrm{~cm}$. Icarus $80,1-13$.

de Pater, I., P. N. Romani, and S. K. Atreya 1991. Possible microwave absorption by $\mathrm{H}_{2} \mathrm{~S}$ gas in Uranus" and Neptune's atmospheres. Icarus 91, 220-233

Dutta, J. M., C. R. Jones, T. M. Goyette, and F. C. Deluicla 1993 The hydrogen and helium pressure broadening at planetary temperatures of the 183 and $380 \mathrm{GHz}$ transitions of water vapor. Icarus 102, 232-239.

Gierasch, P. J., and B. J. Conrath 1987. Vertical temperature gradients on Uranus: Implications for layered convection. J. Geophys. Res. 92, 15,019-15,029.

Goodman, G. C. 1969. Models of Jupiter's Atmosphere. Ph.D. dissertation, University of Illinois, Urbana.

Grizve, A., H. Steppe, D. Graham, and C. J. Schalinski 1994. Disk brightness temperature of the planets at $43 \mathrm{GHz}$ (and $43 \mathrm{GHz}$ flux densities of some continuum sources). Astron. Astrophys. 286, 654-658

GrifFIN, M. J., AND G. S. Or'JoN 1993. The near-millimeter brightness temperature spectra of Uranus and Neptune. Icarus 105, 537-547.

Guilloteau, S., A. Dutney, A. Marten, and D. Gautier 1993. CO in the troposphere of Neptune: Detection of the $J=1-0$ line in absorption. Astron. Astrophys. 279, 661-667.

HoFSTADTER, M. D. 1993. Microwave imaging of Neptune's troposphere. Bull. Am. Astron. Soc. 25, 1077.

Jenkins, J. M., And P. G. Steffes 1988. Constraints on the microwave opacity of gaseous methane and water vapor in the jovian atmosphere. Icarus 76, 378-382.

JoINER, J., AND P. G. STEFFES 1991. Modeling of Jupiter's millimeter wave emission utilizing laboratory measurements of ammonia $\left(\mathrm{NH}_{3}\right)$ opacity. J. Geophys. Res. 96, 17463-17470.

JoINER, J., P. G. STEFFES. AND J. M. Jenkins 1989. Laboratory measurements of the $7.5-9.58 \mathrm{~mm}$ absorption of gaseous ammonia $\left(\mathrm{NH}_{3}\right)$ under simulated jovian conditions. Icarus 81, 386-395.

KING, R. W. P., ANJ G. S. SMITH 1981. Antennas in Matter: Fundamentals, Theory and Applications. MIT Press, Cambridge, MA.

Lindal. G. F. 1992. The atmosphere of Neptune: An analysis of radio occultation data acquired with Voyager 2. Astron. J. 103, 967-982.

LOJDER, K., AND B. FEGLEY, JR. 1994. The origin of carbon monoxide in Neptune's atmosphere. Icarus 112, 368-375.

Lunine, J. 1. 1993. The atmospheres of Uranus and Neptunc. Annu. Rev. Astron. Astrophys. 31, 217-263.

Marten, A., D. Gautier, T. Owen, D. B. Saniders, H. E. Matthews, S. K. Atreya, R. P. J. Tilanus, ani) J. R. Deane 1993. First observations of $\mathrm{CO}$ and HCN on Neptune and Uranus at millimeter wavelengths and their implications for atmospheric chemistry. Astrophys. J. 406, 285-297.

Marten, A., D. Gaither. T. Owfen, D. B. Sanifers, R. P. J. Tilanus, J. R. Deane, and H. E. Mallihews 1991. Bull. Am. Astron. Soc. 23, 1164

Massit, S. T., AND D. M. HUNTEN 1982. Conversion of para and ortho hydrogen hydrogen in the jovian planets. Icarus 49, 213-226. 
Muhleman, D. O., And G. L. Berge 1991. Observations of Mars, Uranus, Neptune, Io, Europa, Ganymede, and Callisto at a wavelength of 2.66 mm. Icarus 92, 263-272.

Orton, G. S., M. J. Griffen, P. A. R. Ade, I. G. Nolt, J. V. Radostitz, E. I. Robson, And W. K. Gear 1986. Submillimeter and millimeter observations of Uranus and Neptune. Icarus 67, 289-304.

Poynter, R. L., ANd H. M. Pickett 1985. Submillimeter, millimeter, and microwave spectral line catalog. Appl. Optics 24, 2235-2240.

Romani, P. N., I. de Pater, and S. K. Atreya 1989. Neptune's deep atmosphere revealed. Geophys. Res. Lett. 16, 933-936.

Rosenquist, J., E. Lellouche, P. N. Romani, G. Paubert, and T. EnCREnaz 1992. Millimeter-wave observations of Saturn, Uranus and Neptune: $\mathrm{CO}$ and HCN on Neptune. Astrophys. J. 392, L99L102.

Sill, G., U. Fink, and J. R. Ferraro 1980. Absorption coefficients of solid $\mathrm{NH}_{3}$ from 50 to $7000 \mathrm{~cm}^{-1}$. J. Opt. Soc. 70, 724-739.
Space Studies Board 1994. An Integrated Strategy for the Planetary Sciences: 1995-2010. National Research Council, Washington, D.C.

SPILKER, T. R. 1990. Laboratory Measurements of Microwave Absorptivity and Refractivity Spectra of Gas Mixtures Applicable to Giant Planet Atmospheres. Ph.D. dissertation, Stanford University, Palo Alto, CA.

Ulaby, F. T., R. K. Moore, and A. K. Fung 1981. Microwave Remote Sensing, Active and Passive, Vol. 1. Artech House, Norwood, MA.

WATERS, J. W. 1976 Absorption and emission of microwave radiation by atmospheric gases. In Methods of Experimental Physics (M. L. Meeks, Ed.), Section 2.3. Academic Press, New York.

Weisstein, E. W., and E. Serabyn 1994a. Detection of the $267 \mathrm{GHz} J=$ 1-0 rotational transition of $\mathrm{PH}_{3}$ in Saturn with a new Fourier transform spectrometer. Icarus 109, 367-381.

Weisstein, E. W., and E. Serabyn 1994b. Detection of the $\mathrm{PH}_{3} J=$ 3-2 rotational transition in Jupiter and Saturn. Bull. Am. Astron. Soc. 26, 1108 . 
